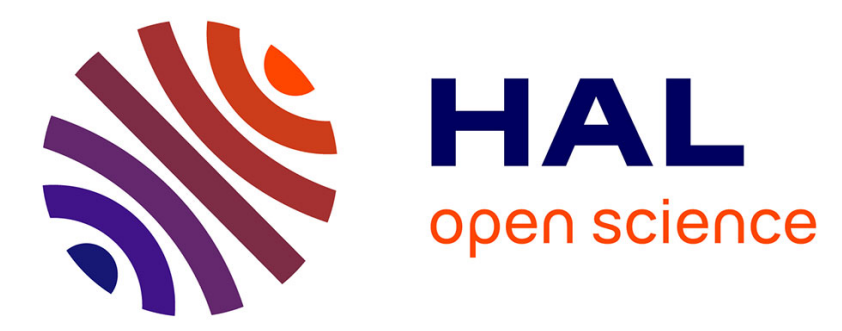

\title{
L'évolution de l'Âge du Bronze Final IIIb et la transition Bronze/Fer en Languedoc oriental d'après la culture matérielle des habitats. Etat des questions.
}

Pierre Garmy

\section{- To cite this version:}

Pierre Garmy. L'évolution de l'Âge du Bronze Final IIIb et la transition Bronze/Fer en Languedoc oriental d'après la culture matérielle des habitats. Etat des questions.. Dialogues d'histoire ancienne, 1979, 5 (5), pp.23-34. halshs-00141433

\section{HAL Id: halshs-00141433 \\ https://shs.hal.science/halshs-00141433}

Submitted on 12 Apr 2007

HAL is a multi-disciplinary open access archive for the deposit and dissemination of scientific research documents, whether they are published or not. The documents may come from teaching and research institutions in France or abroad, or from public or private research centers.
L'archive ouverte pluridisciplinaire HAL, est destinée au dépôt et à la diffusion de documents scientifiques de niveau recherche, publiés ou non, émanant des établissements d'enseignement et de recherche français ou étrangers, des laboratoires publics ou privés. 


\section{L'EVOLUTION DE L'AGE DU BRONZE FINAL IIIb ET LA TRANSITION BRONZE/FER EN LANGUEDOC ORIENTAL D'APRES LA CULTURE MATERIELLE DES HABITATS}

\section{Etat des questions}

Il y a presque vingt ans, paraissait le dernier de trois tomes d'une synthèse due à M. Louis, O. et J. Taffanel (1)* sur ce qu'il était alors convenu d'appeler "Le premier Age du Fer» en Languedoc méditerranéen et Catalogne.

Dans cette étude, qui reste aujourd'hui l'une des références essentielles en la matière, les auteurs distinguaient quatre périodes dont les trois dernières étaient définies par rapport aux séquences de l'oppidum du Cayla de Mailhac et des nécropoles voisines.

La chronologie absolue, comportant trois hypothèses, était établie par récurrence à partir de la quatrième période où apparaissent les premières importations massives du monde méditerranéen.

La tradition a, depuis lors, retenu l'hypothèse moyenne qui est adoptée dans la quasi totalité des écrits postérieurs :

Période 1: ? -750

Période $2: 750$ - 650

Période $3: 650-600$

Période $4: 600$ - 475

Depuis 1960, des fouilles en grand nombre et des approches nouvelles du problème, sans remettre fondamentalement en cause ce premier travail (2), ont permis d'affiner les observations et, en premier lieu, de rendre à l'Age du Bronze les deux premières périodes. Le mérite en revient principalement à $J$. Guilaine qui, dans un ouvrage consacré à l'Age du Bronze en Languedoc occidental (3), distingue, pour ce qui nous concerne ici, les étapes suivantes :

Période 1 : Bronze Final IIIa, "caractérisé par une évolution sur place de la phase méridionale des Champs d'Urnes II", définie au Bronze Final II (p. 304)

Période 2 : «Groupe Mailhac I que l'on peut synchroniser avec le Bronze Final IIIb" et "quelques groupes... qui l'environnent" (p. 305).

Les périodes 3 et 4 de la chronologie Louis-Taffanel inaugurent, dans cette nouvelle optique, le premier âge du Fer, stricto sensu.

*Voir notes, p. 31. 
Tous les archéologues, à l'heure actuelle, à commencer par Mademoiselle Taffanel elle-même, travaillent, en chronologie relative, sur cette grille (4).

Seules intéressent directement notre propos les périodes centrales ( 2 et 3) c'est-à-dire le Bronze Final IIIb et le début de l'Age du Fer jusqu'aux alentours de 600 .

Nous aborderons les problèmes de chronologie absolue en fin d'exposé, à la lumière des recherches récentes.

Le deuxième axe essentiel de la recherche en 1960 concernait le peuplement du Languedoc et de la Catalogue en cette fin de l'Age du Bronze et aux tout débuts de l'Age du Fer.

La théorie, couramment admise à l'époque, sur les traces des chercheurs allemands et en particulier W. Kimmig (5), que l'on retrouve dans de nombreux ouvrages déjà anciens (6) et d'autres beaucoup plus récents (7), considérait le groupe mailhacien I (et les groupes annexes) comme une vague d'envahisseurs venus des régions rhénanes, du Jura souabe et d'Europe centrale, ayant déferlé sur les rivages de la Méditerranée ; la dénomination "civilisation des Champs d'urnes" que l'on rencontre encore dans la littérature archéologique pour désigner les vestiges de la période B.F. IIIb est héritée de cette tradition.

Cette vision a fait long feu, ce pour maintes raisons dont nous rappellerons les principales :

- les séquelles et les jalons de cette progression/invasion géographique sont réellement minces pour ne pas dire inexistantes sur les voies qu'auraient empruntées les "envahisseurs champs d'urnes", sillon Saône-Rhône et façade ouest du Massif Central (8) ;

- l'ensemble du mobilier céramique est en filiation directe avec celui de la période précédente (B. F. IIIa) tant sur le plan des formes élémentaires (voir ci-dessous) que sur celui des motifs décoratifs incisés (9);

- la plus grande partie du mobilier de bronze n'est pas nouvelle : anneaux, bracelets en spirales, épingles à tête plate, sphérique, enroulée, ouvragée sont des héritages des périodes antérieures comme le fait justement remarquer J. Guilaine ;

- les apports étrangers, nombreux et variés, font beaucoup plus appel au monde méditerranéen qu'aux zones d'Europe centrale. Les décors incisés sur céramique, de style zoomorphe et anthropomorphe sont considérés comme d'origine italienne, protovillanovienne ou villanovienne (10). Après N. K. Sandars (11) et J. Guilaine (12) et pour ce qui concerne les bronzes, il serait facile de dresser la liste des discordances entre le groupe alpin et le B.F. IIIb méditerranéen :

- rasoirs circulaires et sub-circulaires, flèches et javelots à pédoncule renflé, fibules à double ressort, tous objets d'origine méditerranéenne, présents dans le midi de la France, sont inconnus sur les stations palafittiques du Bourget, par exemple ;

- inversement le rasoir à tranchant semi-circulaire et manche à anneau, flèches à double perforation sont ignorés sur les rives méditerranéennes ;

- un troisième groupe de bronzes, il est vrai, se retrouve dans les deux horizons mais cela n'a rien d'an ormal, le couloir rhodanien ayant 
dû être à l'époque comme à toute autre, un axe privilégié d'échanges commerciaux.

Il ressort de ce survol rapide que la genèse du B.F. IIIb languedocien et catalan est multiple : elle montre, d'une part, un très fort héritage des périodes immédiatement antérieures (B.F. II et B.F. IIIa) dont les caractères évoluent lentement sur place et, d'autre part, des apports sous forme d'influences, du monde méditerranéen surtout.

Il reste peu de place, dans une telle conception, pour les hordes nordiques ; il est d'ailleurs paradoxal et significatif de lire sous la plume de W. Kimmig (13) : "... ainsi nous imaginons volontiers que la culture des champs d'urnes à été illyrique, dans l'Italie du nord, italique, dans l'espace nord-ouest alpin, celte, en Provence, peut-être ligure, en Espagne même, ibérique...».

Il est également d'usage d'expliquer la période suivante (période III de Louis-Taffanel) par une nouvelle invasion sur l'origine de laquelle les avis divergent largement : ibérique pour $J$. Jannoray, nord-alpine à nouveau pour d'autres ou inconnue. $\mathrm{Si}$, comme nous venons de le rappeler, on peut aisément faire l'économie d'une telle hypothèse pour la période II, les réponses ne sont pas, dès l'abord, aussi évidentes pour ce qui suit. Nous essayerons d'avancer, en fin d'exposé, quelques orientations.

Tout ce que nous venons d'énoncer ou de rappeler a été, pour une bonne part déduit des observations faites en Languedoc occidental et en Catalogne.

En effet, jusqu'à une date récente, l'Age du Bronze Final IIIb et les tout débuts de l'Age du fer en Languedoc oriental étaient particulièrement mal connus. Seuls deux sites de ces époques sont mentionnés dans l'ouvrage de Louis-Taffanel (Roque de Viou et Languissel).

Plus récemment, J. L. Roudil, dans un livre (14) faisant pendant, pour la partie orientale du Langue doc à celui de J. Guilaine, considère les témoins de ce type comme intrusifs : "Le mailhacien a, en effet, essaimé d'ouest en est dans le sud de l'Hérault, mais au delà de Montpellier ses traces deviennent très sporadiques» (15).

Pourtant, dès 1963, H. Gallet de Santerre (16) prévoyait, de manière logique, la prochaine découverte de gisements de cette période dans le Gard. Cette prédiction a été largement confirmée par les faits puisqu'une douzaine de sites ont été depuis lors découverts et exploités (17) dont nous nous proposons d'étudier ici les caractères généraux par ordre chronologique.

\section{Le Bronze Final IIIb :}

Le mobilier de cette période constitue un tout parfaitement uni et reconnaissable. $\mathrm{La}$ culture matérielle est essentiellement représentée par la céramique, qui constitue 80 à $90 \%$ du matériel recueilli dans chaque fouille, et le mobilier métallique. Celui-ci est très peu abondant en Languedoc oriental, contrairement à la partie occidentale.

Ce sont surtout des épingles dont la tête a des formes très variées : vasiformes (Bergerie Hermet et Gauto Fracho), arrondies et souvent moulurées (Gauto Fracho et Roque de Viou) et d'autres exemplaires incomplets (Roc de Gachonne) ; des anneaux (Roc de Gachonne et Triple Levée) et une pointe de flèche triangulaire à pédoncule renflé (découverte fortuitement à Caveirac, Gard, après les labours saisonniers). Il semble que cette pauvreté générale en matériel de bronze soit due, en grande partie à l'absence de grandes nécropoles dans notre région. 
Le seul type céramique connu à l'époque est la céramique modelée (18). Les techniques de cuisson sont encore très archaïques : seules semblent u tilisées les cuissons en meules et en fosses qui ne permettent pas de maîtriser les phases d'oxydation, de réduction et combinées, comme en témoignent les couleurs très variées que montrent les pâtes et les épidermes de la plupart des vases (19).

- Les urnes présentent la forme type suivante (20) : panse arrondie très galbée, fond plat ou légèrement creux, le plus souvent à base élargie, col évasé, équipé soit d'un bord dans l'axe du col, soit d'un bord déversé, muni d'une à trois facettes ou méplats.

Généralement, le haut de la panse ou épaulement est monté en méplats et le plus souvent décoré de divers motifs : cannelures, impressions diverses et plus rarement incisions.

La surface extérieure des urnes est presque toujours mieux aménagée que la surface intérieure, ce qui n'a rien que de très normal pour un vase fermé.

- Les coupes, quant à elles, sont de trois formes principales :

Les coupes bitronconiques à carène, de dimensions très variables ; munies d'un fond plat ou creux, une vasque plus ou moins ouverte et un bord rentrant de profil simple. La force et l'emplacement du changement de courbure varient d'un exemplaire à l'autre.

Cette forme est, avec la suivante, le support privilégié des décors incisés dont il sera question plus loin.

Les coupes et assiettes tronconiques sont facilement reconnaissables et très fréquentes au B. F. IIIb : coupes très ouvertes, à fond le plus souvent creux ou annulaire, à bord fréquemment déversé et faceté.

Les aménagements de surfaces sont très typiques : l'extérieur est laissé brut de montage ou grossièrement lissé alors que l'intérieur a retenu tous les soins du potier : polissage ou lissage très soigné sur lesquels on implante ensuite, dans de nombreux cas, des décors de cannelures, d'incisions ou des décors peints.

Ces deux premières formes de coupes, bitronconiques et tronconiques, sont spécifiques de cette époque.

La dernière forme de coupe est aussi la plus simple : de forme subhémisphérique à fond plat ou creux et bord évasé.

La surface intérieure en est presque toujours mieux aménagée que la surface extérieure.

Les décors, de plusieurs types, requièrent des tech̆niques diverses :

- les cannelures héritées des périodes précédentes mais qu'on ne trouve plus, au B.F. IIIb que sous forme concentrique et horizontale (exceptionnellement en chevrons). Leur implantation préférentielle est située sur les épaulements, sur les lèvres et autour du fond des assiettes tronconiques ;

- les impressions diverses (21) obtenues par enfoncement d'un objet dans la pâte fraîche. Situés presqu'uniquement sur les épaulements d'urnes, parfois en association avec les cannelures, ces décors sont de formes et de dimensions très variées. Par combinaison, le potier a donné sur certains vases des compositions complexes ;

- les incisions en double trait qui représentent la technique la plus spécifique de ces périodes de la fin de l'âge du Bronze, surtout sous leurs formes anthropomorphes et zoomorphes. Ce sont de fines déchirures de l'épiderme des vases, qui peuvent être remplies de matière colorée blanche ou 
rouge (22). Le répertoire connu regroupe les formes suivantes : géométriques (simples lignes horizontales, lignes brisées et chevrons, méandres et grecques plus ou moins compliqués et triangles hachurés), zoomorphes («petits chevaux» très stylisés) et anthropomorphes (23).

Tous ces motifs incisés peuvent entrer en composition pour former des sortes de «processions» ou «danses», des «attelages» où la roue est représentée par un rectangle et ses diagonales (24).

Les décors incisés se rencontrent surtout sur la face extérieure des coupes bitronconiques, au dessus de la carène en un ou plusieurs registres et dans la vasque des assiettes tronconiques, de la lèvre jusqu'au fond, soit en registres concentriques soit en métopes rayonnantes.

- Certains vases enfin sont peints, en tout ou partie, de rouge sombre.

Ces descriptions sont d'ordre global, pour l'ensemble du B. F. IIIb, mais le faciès du mobilier en Languedoc oriental laissait apparaître des différences notables d'un site à l'autre et une durée certaine, en contradiction avec l'absence de stratification interne des gisements, jusqu'à une date récente.

Les dernières années ont apporté une moisson de nouvelles informations, principalement sur trois sites qui ont permis de mettre en évidence une évolution typologique et stylistique du mobilier : il s'agit des gisements lagunaires du littoral héraultais (inédit), de Roque de Viou (fouille RE 12) (25) et du Roc de Gachonne (26), deux habitats de hauteur de la région nîmoise.

Les trois habitats renfermaient les couches stratifiées de plusieurs occupations successives appartenant toutes à la période qui nous intéresse et ont montré que l'évolution se faisait partout de manière continue et univoque.

Les formes céramiques se transforment dans le temps selon un schéma que l'on commence à saisir ; il n'est pas question, ici, d'entrer dans les détails, rappelons seulement les voies générales (27).

La tendance principale est à un amollissement génêral des profils de la vaisselle : le contact entre les diverses parties du vase est moins marqué, les lèvres s'arrondissent, les fonds à base élargie disparaissent petit à petit, les carènes s'adoucissent, etc...

On assiste également à un phénomène de substitution de certaines formes à d'autres : les vases à embouchure rétrécie, par exemple, augmentent en nombre au détriment des coupes bitronconiques.

La décoration des vases s'appauvrit et le répertoire s'épuise : disparition progressive des motifs zoomorphes et anthropomorphes, facture de moins en moins bonne (apparition des lignes courbes jusqu'alors bannies), si bien que l'on peut distinguer, en association avec une étude typologique précise, un style sévère, comportant l'ensemble du répertoire et de bonne facture et un style tardif ayant abandonné certains types et dont l'exécution laisse à désirer. Les incisions apparaissent, dans cette phase récente sur des formes où l'on n'avait pas l'habitude de les rencontrer, notamment sur des coupes subhémisphériques.

La plus extrême prudence est encore de mise quand il s'agit de tirer les conclusions possibles de ces constatations. Ont certainement joué, ensemble, des facteurs chronologiques et géographiques, dans une mesure qu'il est encore difficile de déterminer. Il est cependant intéressant de constater que, sur tous les sites de la frange rhodanienne et des confins septentrionaux des garrigues nîmoises, le style sévère n'est pas attesté : il y a là, soit des gisements sans motifs zoomorphes et anthropomorphes, soit d'autres où ceux-ci sont présents mais très évolués (voir, par exemple le motif que d'aucuns ont appelé "oiseau» sur une coupe de Triple Levée à Beaucaire). 
Franchissant le Rhône, les mêmes remarques s'imposent pour les sites de Provence occidentale qui semblent appartenir dans leur totalité à une phase tardive du B. F. IIIb (28) de laquelle pourraient relever la Balance à Avignon (29), la Grotte basse de Vidauque (30) Glanum à St Rémy-deProvence (31) et de petits sites comme Espuy à Venasques (inédit) ou du Mont de Cordes à Fontvieille (32). Les vestiges du Pègue (33) paraissent encore plus récents ce qui peut s'expliquer par la position très reculée du site, de même pour le site de Moras-en-Valloire (34).

La chronologie des vases à couverte peinte en rouge sombre n'est pas très assurée : de manière générale l'ensemble de la production semble tardif (35).

Les débuts du Premier Age du Fer :

Les habitats de la période III de la chronologie Louis-Taffanel semblaient jusqu'à ces dernières années absents du domaine languedocien oriental.

On n'avait, jusqu'alors, aucun indice qui permette de penser qu'il y ait eu entre Hérault et Rhône la phase que représente à Mailhac et alentour le faciès Grand Bassin I. Cela ne présentait d'ailleurs rien de surprenant tant il semble qu'au regard de l'évolution céramique, on puisse «logiquement» faire l'économie de cette phase intermédiaire. En effet, toutes les tendances que nous décrivions plus haut se perpétuent en descendant le temps, de manière continue dans le plein VIe siècle (et même après).

De prime abord, tout concorde pour faire de ce faciès de la fin VIIe siècle un "accident génétique» ou un élément intrusif. Ces remarques valent également pour la région de Mailhac où il est surprenant de constater que la céramique modelée du Grand Bassin II, par exemple, est en filiation directe de celle du Moulin, selon les schémas énoncés plus haut (36) par delà l'épisode Grand Bassin I.

Donnons rapidement l'inventaire des trouvailles de cette époque à l'est de l'Hérault : il s'agit d'est en ouest des habitats de plein air de Port-Vielh près d'Aigues-Mortes (37), de la Liquière à Calvisson (38), de la Redoute à Beaucaire (39) et de l'occupation de la grotte Suspendue à Collias et de quelques grottes de la vallée du Gardon (40).

Sur tous ces sites, la céramique modelée est encore très largement prédominante, voire même la seule représentée.

- Les urnes sont de deux sortes :

* la première, minoritaire mais très spécifique du faciès consiste en vases à grand col en calice portant une lèvre à méplats, une panse carénée et un pied plus ou moins haut. Certaines de ces pièces sont décorées sur le col et l'épaulement de faisceaux d'incisions en chevrons, de cannelures et d'impressions diverses, d'autres d'excisions ;

* la seconde est directement issue des formes du B. F. IIIb dont elle représente un type évolué à panse ovoïde : vases à col court, le plus souvent à bords parallèles et sans lèvre distincte, à contact col-panse marqué faiblement ou pas du tout et très peu galbé. ment :

- Les coupes sont simples et de formes moins variées que précédem-

* la plupart a un profil arrondi-convexe qui se referme légèrement à l'ouverture, un fond plat ou annulaire ;

* certains exemplaires présentent, à l'intérieur de la lèvre, un petit décroche- 
ment de la paroi, trait typique de ce faciès. On rencontre également quelques coupelles subhémisphériques à fond creux, parfois décorées d'excisions, et des formes tardives de la coupe bitronconique du Bronze Final.

- A cette époque encore, les habitats sont pauvres en mobilier métallique ; on note cependant l'apparition d'objets en fer (petite hache à douille ronde sur l'oppidum de la Liquière) à côté du mobilier de bronze qui reste très présent : fibule serpentiforme notamment.

On voit, par les descriptions qui précèdent, que bon nombre d'éléments ne sont pas des innovations complètes de l'époque, mais invoquer un simple héritage des périodes antérieures ne suffit pas à tout expliquer de ce faciès complexe (41).

La datation absolue est facilitée par la présence, sur deux sites, Grotte suspendue et la Liquière, d'importations méditerranéennes, en connexion avec les céramiques modelées de notre groupe.

Il s'agit dans le premier cas d'un skyphos de type rhodien à bandes, bien connu en Grèce de l'est et dans les colonies ioniennes et dont on a trouvé six autres exemplaires en Gaule du sud (tumulus de Claps près d'Aix en Provence, La Couronne à l'Arquet, St Blaise et Marseille (42), la Rallongue (43) et la Monédière à Bessan (44), tous datables du dernier quart VIIe ou des premières années du Vle siècle avant notre ère.

Dans le deuxième cas, on a affaire à du matériel essentiellement étrusque : amphores vinaires de type 1 et 2 (45) et canthares en bucchero nero de modèles anciens (incisés sous la lèvre), trouvés immédiatement au dessous d'une couche contenant des tessons de corinthien ancien (46) et des coupes ioniennes A 1 (47). Cette deuxième série de documents correspond chronologiquement à la date donnée pour le skyphos rhodien (48).

$\mathrm{Vu}$ la grande homogénéité de faciès que présentent tous les sites, il semble qu'on doive leur assigner comme datation la deuxième moitié ou peut-être même le dernier tiers du VIIe siècle avant notre ère.

La «parenté» de ce groupe oriental avec les vestiges similaires de l'ouest de l'Hérault est évidente. On insistera sur les comparaisons qui peuvent être faites d'élément à élément entre les deux domaines. La similitude du mobilier métallique, pour ce qu'on en connaît à l'heure actuelle renforce cette ressem. blance générale tant chronologique que du faciès de la culture matérielle. Seuls manquent, dans le domaine rhodanien, les influences puniques que l'on trouve à Mailhac et d'autres sites de la région, mais c'est une constante de toute la protohistoire languedocienne.

La date exacte et les modalités d'apparition de ce faciès sont encore peu claires, mais on peut d'ores et déjà souligner les faits suivants :

- à la Redoute à Beaucaire, la couche 3 b du sondage 3, qui intéresse directement notre propos, surmonte immédiatement la couche 4 ressortant de la phase tardive du B.F. IIIb ;

- à Mailhac, le passage de la nécropole du Moulin à la nécropole du Grand Bassin I se fait sans heurt. Les tombes les plus tardives de la première (quatre dans l'état actuel des fouilles (49) présentent : - une céramique qui appartient strictement à la période II, mais aussi - les premiers objets en fer et les caractères d'organisation de la sépulture type de la période III (nombre de vases, dépôts, taille du loculus...)

Ceci fait :

- que nous ne concevons pas, à l'heure actuelle, la possibilité d'intercaler entre la fin du B.F. IIIb et ce groupe fin VIIe, un faciès intermédiaire; 
- que le passage de l'un à l'autre ne marque pas une réelle rupture brusque même si des éléments incontestablement étrangers viennent se greffer sur le substrat en place.

De toutes ces constatations et réflexions, il demeure que pour l'essentiel, la chronologie relative et absolue proposée par Louis-Taffanel en 1960 reste valable à la lumière des recherches les plus récentes même si l'on doit remonter le terminus post quem de la période II au regard d'une durée certaine du B. F. IIIb (50).

Il semble également qu'il faille définitivement substituer à la notion de «vagues d'envahisseurs» successives celle d'apports économiques et culturels permanents venant s'ajouter à un fond de civilisation stable et qui assimile petit à petit ces influences. Les apports ethniques, s'il y en a eu, ont été faibles et n'ont pas laissé de marques durables sur l'évolution des cultures matérielles autoch tones.

Les recherches à venir devront aider à identifier les processus et l'impact exacts de tous ces apports sur le milieu ambiant et éclaircir nombre de points encore obscurs.

\section{ADDENDA}

Les problèmes concernant les sépultures et le mobilier funéraire dépassent les limites de notre propos. Il semble néanmoins indispensable de signaler brièvement les principales voies de la recherche récente dans ce domaine.

- Le Languedoc oriental, comme nous l'avons déjà dit, n'a livré pour le moment aucune nécropole ou sépulture du B. F. IIIb hormis une tombe sous tumulus, à la limite du domaine languedocien, dans le sud de l'Ardèche (51).

- A la période suivante, par contre, appartiennent de nombreux tumulus du Gard et de l'Hérault. Ceux-ci, dans les conceptions de Louis-Taffanel, s'inscriraient dans le cadre de la "civilisation des tumulus", dont le domaine géographique s'étend sur la bande de garrigues septentrionales, alors que les plaines littorales seraient occupées par une "civilisation des champs d'urnes" (52), ce schéma étant appliqué au Languedoc occidental (où l'on ne connaît pas de tumulus) et au Languedoc oriental (où il n'y a pas, à l'heure actuelle de nécropoles à incinération). La carte des gisements établie par ces auteurs (53) vient d'ailleurs en parfaite contradiction avec ces hypothèses.

B. Dedet vient, à ce sujet, de livrer un travail de réflexion fondamental (54) qui remet complètement en cause à la fois la répartition nord-sud des deux groupes (pour établir une coupure est-ouest qui est nette sur la carte précitée) et la dichotomie que l'on voulait établir de l'un à l'autre au vu du mobilier de chacun. Il affirme également la grande ressemblance pour ne pas dire l'identité du matériel (métallique et céramique) exhumé dans les tumulus et les habitats contemporains du Languedoc oriental, qui répond, ici et là, aux descriptions que nous donnions ci-dessus.

De là ressort que rites funéraires et culture matérielle ne sont pas indissolublement liés puisque des restes très voisins ont été recueillis de chaque côté de l'Hérault, en Languedoc occidental dans des nécropoles à incinération et en Languedoc oriental dans les tumulus.

Un travail très documenté, dû à Y. Gasco (55), donne un inventaire 
complet des principaux tumulus languedociens pour lesquels les publications an térieures étaient très partielles et lacunaires.

\section{Pierre GARMY}

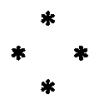

NOTES

(1) M. LOUIS, O. et J. TAFFANEL, Le premier Age du Fer languedocien, I Les habitats, II Les nécropoles à incinération, III Les tumulus, conclusions, Bordhigera-Montpellier, 1955,1958 et 1960.

(2) Un récent travail d'une équipe italienne, dirigée par Monsieur R. PERONI, confirme, pour l'essentiel, la chronologie relative des champs d'urnes languedociens : Sulla cronologia dei campi di urne della Linguadoca, Rivista di scienze prehistoriche, XXXI, 1, Firenze 1976.

(3) J. GUILAINE, L'Age du Bronze en Languedoc occidental, Roussillon, Ariège, Mémoires de la S.P.F., no 9, 1972.

(4) Voir, par exemple le compte rendu d'une réunion tenue à Sète le 8 juin 1975 dans Fédération archéologique de l'Hérault, Le Languedoc au Premier Age du Fer, notamment p. 10.

(5) W. KIMMIG, Zur Urnenfelderkultur in Südwesteuropa, Festschrift für Peter Goessler, Stuttgart 1954. Du même, Où en est l'étude de la civilisation des Champs d'Urnes en France, principalement dans l'Est ?, RAE, II, 1951 p. 65-81, III, 1952 p. 7-19 et 137-172, V, 1954 p. 7-28 et 209-232.

(6) M. LOUIS O et J. TAFFANEL, Le premier Age..., op. cit., et J. JANNORAY, Ensérune, contribution d l'étude des civilisations préromaines de la Gaule méridionale, Paris, 1955.

(7) M. CLAVEL, Béziers et son territoire dans l'Antiquité, Paris 1970.

(8) Certains auteurs ont voulu tirer argument de la position très septentrionale de certains sites comme l'oppidum de Fort Harrouard à Dreux (LOUIS-TAFFANEL, I p. 174), la nécropole de Villement à St Aoustrille, dans l'Indre (ibid. p. 175) et de découvertes plus récentes en Charente, La grotte de Cheroua à Chazelles (Actes du $3^{e}$ colloque de préhistoire et d'archéologie du Libournais, RHAL, XLII, 1974) et dans la Vienne, Camp Allaric (J.-P. PAUTREAU, Un vase hallstatien à décors anthropomorphes au camp Allaric, commune d'Arlonnes, Vienne, et l'annexe qui suit, Bulletin de la S.P.F. no 69-7, 1972) ; il est à remarquer que, d'une part le synchronisme n'est pas établi avec les sites méridionaux et que, d'autre part, la plus extrême prudence s'impose pour parler de faciès qui doit être étudié d'après une typologie précise et non à partir de ressemblances du répertoire décoratif.

(9) Le répertoire géométrique du B. F. Illb est déjà connu et employé antérieurement et en particulier le motif en méandres et grecques (méandre en $\mathrm{H}$ couché notamment). A ce propos, on verra R. ARIS et J.-J. JULLY, A propos d'un fragment de céramique inédit à décor incisé provenant de Cessero (St Thibéry, Hérault), CLPA no 17,1968 , p. $81-101$, très abondante et complète bibliographie et $\mathrm{J}$. ARNAL et A. SOUTOU, La céramique incisée à méandres symétriques du Pont du Diable, Aniane, Hérault, Gallia 19, 1961, p. 201-218. 
(10) M. LOUIS, O. et J. TAFFANEL, Le premier Age... III, op. cit., p. 335 et H. MULLER KARPE, Beitrage zur chronologie der Urnenfelderzeit nördlich und südlich der Alpen, Berlin 1959, et J. GUILAINE, L'Age du Bronze..., op. cit. N. K. SANDARS, Bronze Age culture in France, Cambridge 1957.

(13) W. KIMMIG, Zur Urnenfelderkultur..., op. cit.

(14) J.-L. ROUDIL, L'Age du Bronze en Languedoc oriental, Mémoires de la S.P.F., no $10,1972$.

(15) Ibid. p. 140, cité par B. DEDET, L'habitat de hauteur du Grand Ranc à Boucoiran (Gard) et le Bronze Final IIIb dans les garrigues du Languedoc oriental, Gallia Préhistoire 21, 1978 - 1.

(16) H. GALLET DE SANTERRE, Le problème des champs d'urnes dans le Gard, Bulletin de la société d'histoire et d'archéologie de Beaucaire, 7, 1963.

(17) Il s'agit, d'ouest en est des habitats de Roque de Viou à St Dionisy (voir en dernier lieu P. GARMY et M. PY, Deux cabanes stratifiées de l'Age du Bronze Final IIIb sur l'oppidum de Roque de Viou à St Dionisy (Gard), Gallia Préhistoire 19, 1976 - 1, dans lequel on trouvera la bibliographie antérieure), la Bergerie Hermet à Calvisson (P. GARMY, Un village de l'Age du Bronze Final IIIb à la Bergerie Hermet (Calvisson, Gard), à paraître), le Roc de Gachonne à Calvisson (P. GARMY, Premières recherches sur l'oppidum du Roc de Gachonne à Calvisson (Gard), à paraître), Languissel, près de Nîmes (C. HUGUES, Céramiques des champs d'urnes des plaines et des oppida du Gard, Celticum VI, 1963), le Grand Ranc à Boucoiran (B. DEDET, L'habitat de hauteur du Grand Ranc..., op. cit), Gauto Fracho à Bouquet (B. DEDET, L'oppidum de Gauto Fracho à Bouquet (Gard), $R A N$. VIII, 1975), Triple Levée et La Redoute à Beaucaire (B. DEDET, A. MICHELOZZI, M. PY, C. RAYNAUD et C. TENDILLE, Ugernum, protohistoire de Beaucaire, $A R A L O$, cahier no 6, 1978). Le Marduel à Remoulins (inédit), les grottes de la vallée du Gardon (A. COSTE, B. DEDET, X. GUTHERZ et M. PY, L'occupation protohistorique de la grotte suspendue à Collias, Gard, dans son contexte des Gorges du Gardon, Gallia, 34, 1976), la Combe des Charlots à Sainte Anastasie (inédit) et Montaillon à Sanilhac-et-Sagriès (inédit).

(18) Sur cette notion et les principes de fabrication, on consultera avec profit $G$. CAMPS, Monuments et rites funéraires protohistoriques, aux origines de la Berbérie, Arts et Métiers graphiques, Paris, 1961, deuxième partie.

(19) M. PICON, Introduction d̀ l'étude technique des céramiques sigillées de Lezoux, Centre de recherches sur les techniques gréco-romaines, no 2, 1973 et Les dossiers de l'Archéologie, Les potiers gaulois, no 6, 1974.

(20) On trouvera l'illustration nécessaire dans la plupart des monographies citées plus haut (note 17) et également dans l'ouvrage de B. DEDET et M.PY, Introduction d l'étude de la Protohistoire en Languedoc oriental, ARALO, cahier no 5, 1976.

(21) Le site de la Bergerie Hermet a livré un corpus très complet de ce type de décors : P. GARMY, Un village..., op. cit.

(22) P. GARMY, A propos d'un vase de l'oppidum de Roque de Viou (Gard) : observations sur la technique des décors incisés du Mailhacien I, Études préhistoriques no $9,1974$.

(23) J. GUILAINE, L'Age du Bronze..., op. cit., p. 325.

(24) La nécropole de Vendres (Hérault) renferme un vase portant une composition de ce type particulièrement riche. Voir : P. ABAUZIT, Nécropole du Premier Age du Fer à Vendres. Hérault, BSPF LVIII, 1961, 3-4.

(25) P. GARMY et M. PY, Deux cabanes stratifiées..., loc. cit.

(26) P. GARMY, Premières recherches..., loc. cit.

(27) Pour plus de détails sur les méthodes statistiques utilisables en ce domaine, lire 
B. DEDET, A. MICHELOZZI, M. PY, ..., Ugernum..., op. cit., p. 71 sq. ; B. DEDET, L'habitat de hauteur du Grand Ranc..., loc. cit. et P. GARMY, Premières recherches..., loc, cit.

(28) Bien qu'aucune étude précise n'en ait encore été faite, nous fondons ces réflexions uniquement sur des critères stylistiques.

(29) S. CAGNIÈRES et J. GRANIER, Avignon de la préhistoire à la papauté, Avignon, 1971 et exposition permanente du musée Calvet, salles de préhistoire.

(30) M. LOUIS, O. et J. TAFFANEL, Le Premier Age..., op. cit., p. 64.

(31) H. ROLLAND, Nouvelles fouilles du sanctuaire des Glaniques, RSL, 34, 1968 (Hommage d F. Benoit 11,1972, p. 9 à 13 et fig. 2 à 7 ).

(32) R. MONTJARDIN, Quelques vestiges du Bronze Final au Premier Age du Fer, Ogam 15, 1963.

(33) C. LAGRAND et J.-P. THALMANN, Les habitats protohistoriques du Pegue (Dróme), le sondage 8, Grenoble 1973, p. 120-121.

(34) A. NICOLAS, Le site de Moras-enValloire, Archéologia, $65-1973$, p. 30 à 38 ; et A. NICOLAS et B. MARTIN, La céramique incisée de Moras-en-Valloire (Drôme), Etudes préhistoriques no 2, 1972 et les annexes de J.-L. ROUDIL et J. COMBIER qui suivent.

(35) Ces vases sont signalés au Pègue (LAGRAND-THALMANN, op. cit., p. 41, note 46), dans un tumulus et un habitat en grotte associés à des céramiques de type Bronze Final et à des tessons de corinthien ancien de la fin VII $:$ Tumulus de Pourrières, cité dans F. BENOIT, Recherches sur l'hellénisation du midi de la Gaule, Aix en Provence 1965, p. 171 et note 73, et grotte de Baudinard, Gorges du Verdon, LAGRAND, CLPA $196211 / 2$ p. 263 et CLPA $196312 / 2$ p. 251, 252 ; on connaît également des exemplaires polychromes sur les stations du type du Lac du Bourget (Savoie) : Station de Grésine, Gallia XIX, 1961 p. 211 fig. $\left.12 n^{\circ} 7\right)$ et Sassenage, A. BOCQUET, Une station protohistorique à Sassenage (Isère), BSPF LXIII 1966. Les seuls exemplaires connus sur des sites éventuellement plus anciens ont été recueillis sur l'habitat du Roc de Gachonne, P. GARMY, Premières recherches... loc. cit.

(36) Les urnes, particulièrement, sont des formes évoluées de celles du Moulin.

(37) X. GUTHERZ et M. PY, Note sur l'habitat protohistorique de Port-Vielh à Aigues Mortes (Gard), RAN IX 1976, p. 191 à 201.

(38) M. PY, Les oppida de Vaunage (Gard) Fouilles 1958-1968, Thèse de doctorat de III e cycle, Montpellier 1972, dactylographiée ; M. et F. PY, Les amphores étrusques de Vaunage et de Villvieille (Gard), MEFRA tome 68, 1974-1.

(39) B. DEDET, A. MICHELOZZI, M. PY..., Ugernum..., op. cit., p. 64, 65 sq.

(40) A. COSTE, B. DEDET, X. GUTHERZ et M. PY, L'occupation protohistorique de la Grotte suspendue de Collias (Gard) dans son contexte des gorges du Gardon, Gallia, 341976.

(41) Une fouille récente en Provence occidentale a mis au jour des vestiges comparables, voir : P. ARCELIN et J. BREMONT, Le gisement protohistorique du Mont Valence (Fontvieille, Bouches du Rhône), Cypsela II, 1978, sous presse. Le matériel tourné se compose d'une anse d'amphore étrusque (pâte de type $3 \mathrm{Al}$ ) et un bord de coupe d'imitation régionale de type $B 1$ (semblable à celui qu'a livré le gisement de La Liquière dans sa phase I récent).

(42) La bibliographie concernant ces découvertes est donnée par A. COSTE, B. DEDET, X. GUTHERZ et M. PY, L'occupation protohistorique..., loc. cit., p. 153. Voir également la discussion sur les dates de ces importations dans : J.-P. MOREL, L'expansion phocéenne en Occident, dix années de recherches 1966-1976, BCH, XCIV, 1975.

(43) G. MARCHAND, Importations de céramiques grecques archaiques sur le littoral 
lagunaire des environs de Montpellier, Gallia 36, 1978, p. 1-18.

(44) J.-J. JULLY, Coupe rhodienne de la Monédière (Bessan), Hérault, et comparaisons, $R A N 10,1977$ p. $219-221$.

(45) M. et F. PY, Les amphores étrusques... loc. cit.

(46) F. PY, La céramique corinthienne de La Liquière (commune de Calvisson, Gard) et son interprétation, RSL (Hommage d F. Benott $\mathrm{l}, \mathrm{p} .277$ et sq.).

(47) G. VALLET et F. VILLARD, Megara Hyblaea V, lampes du VIIe siècle et chronologie des coupes ioniennes, MEFRA LXVII, 1955, p. 7-24.

(48) Au Grand Bassin I à Mailhac, on a trouvé, au dessus d'une tombe deux éléments de coupes dites de Thapsos, que l'on date couramment de la deuxième moitié VIIe, ce qui établit un élément de synchronisme avec les gisements orientaux.

(49) Aimable renseignement de Mademoiselle O. TAFFANEL.

(50) Nous ne suivrons pourtant pas J. GUILAINE pour monter si haut qu'il le fait ce terminus, L'Age du Bronze..., op. cit., p. 204 sq., pas plus d'ailleurs que le raisonnement de Mademoiselle $O$. TAFFANEL sur la durée d'utilisation de la nécropole du Moulin, bâti sur des a priori non vérifiables, Chronologie du Bronze Final dans l'Aude d'après la nécropole du Moulin à Mailhac, $18^{e}$ congrès UI.S.P.P., Colloque $X X V$, Les champs d'urnes dans le midi de la France, Nice 1976, p. 11-20.

(51) O. et A.-C, GROS, Le tumulus protohistorique no 1 de l'Abeillou à Grospierres, Etudes préhistoriques, no 2, p. 15 à 19.

(52) M. LOUIS, O. et J. TAFFANEL, Le premier Age.., op. cit., III Les Tumulus, conclusions.

(53) Ibid. carte hors texte.

(54) B. DEDET, Les tombes du Languedoc oriental au Premier Age du Fer dans leur contexte culturel : acquis et problèmes, à paraître dans RAN XII, 1979.

(55) Y. GASCO, Les tumulus du Premier Age du Fer en Languedoc oriental, thèse de doctorat de III' cycle, Montpellier 1978, dactylographiée. 